\title{
Detection and tumour grading of prostate cancer in community hospitals
}

\author{
Wassim Kassouf, MD
}

See related article on page 518

S everal studies have examined the variations between community pathologists and urological pathologists with respect to the detection and Gleason grading of prostate cancer via transrectal ultrasound-guided core biopsies of the prostate gland. ${ }^{1}$ Most studies are based on American and European hospitals, thus I congratulate Newell and colleagues ${ }^{2}$ on their study examining these differences in the Canadian population.

The authors found a significantly higher rate of categorical diagnosis of prostate cancer at prostate gland biopsy in community hospitals than in university hospitals (58\% v. 33\%). Detection secondary to casefinding can be more evident in a community where prostate cancer screening may have been delayed and can explain the higher detection rates of prostate cancer found in their study. Several other factors are known to influence the yield of prostatic biopsy in the detection of cancer, including serum prostate-specific antigen (PSA) levels, findings from digital rectal examinations (induration v. hard nodules) and age. We also know that the size of the prostate may be a factor influencing prostate cancer detection rates. In Canada, where health care is provided in a single-payer system with limited resources, community urologists may have a different threshold for biopsy compared with university urologists.

Furthermore, Newell and colleagues found significant variations among community pathologists in the diagnosis and grading of prostate cancer at biopsy. Previous studies have also shown that community pathologists tend to "undergrade" prostate cancer found at biopsy. ${ }^{1}$ In the present study, the incidence of Gleason score 6 prostate cancer was 36\% compared with $49 \%$ in the literature; however, without review of all prostate gland biopsies by a dedicated urological pathologist, it is not possible to determine whether community pathologists in Canada also "undergrade" prostate cancer. Factors such as the size of the prostate and the extent of prostatic biopsies have been recently shown to influence Gleason scores and the incidence of "upgrading" on prostatectomy specimens; these factors should be taken into account when evaluating this specific issue. ${ }^{3}$ In larger glands, elevated serum PSA levels (the driving factor for a biopsy) are commonly the result of benign prostatic hyperplasia that is concomitantly present when cancer is found. The cancers detected in this setting typically can be well differentiated and low in volume. I believe it is beneficial to obtain a second consultation with a urological pathologist - especially for patients with prostate cancer who are being considered for active surveillance - to ensure the absence of higher-grade disease.

From the Division of Urology, McGill University Health Centre, Montréal, Que.

Competing interests: None declared.

\section{References}

1. Steinberg DM, Sauvageot J, Piantadosi S, et al. Correlation of prostate needle biopsy and radical prostatectomy Gleason grade in academic and community settings. Am J Surg Pathol 1997;21:566-76.

2. Newell KJ, Amrhein JF, Rashmikant J, et al. Prostate gland biopsies and prostatectomies: an Ontario community hospital experience. CUAJ 2008;2:518-23.

3. Kassouf W, Nakanishi H, Ochiai A, et al. Effect of prostate volume on tumor grade in patients undergoing radical prostatectomy in the era of extended prostatic biopsies. J Urol 2007;178:111-4.

Correspondence: Dr. Wassim Kassouf, Division of Urology, McGill University Health Centre, 1650 Cedar Ave., Rm. L8-315, Montréal QC H3G 1A4; wassim.kassouf@muhc.mcgill.ca 